\title{
Experimental Results with Forward Erasure Correction and Real Video Streaming in Hybrid Wireless Networks ${ }^{1}$
}

\author{
Paolo Barsocchi ${ }^{\circ}$, Alberto Gotta ${ }^{\circ}$, Francesco Potortì ${ }^{\circ}$, \\ Francisco J. González-Castaño", Felipe Gil-Castiñeira", \\ Jose I. Moreno, Antonio Cuevas ${ }^{\S}$ \\ ${ }^{\circ}$ ISTI-CNR, Area della Ricerca del C.N.R., Via Moruzzi 1, I-56124 Pisa, Italy. \\ \{paolo.barsocchi,alberto.gotta,Potorti \}@isti.cnr.it \\ "Dpto. de Ingeniería Telemática, Universidad de Vigo, ETSI Telecomunicación, Campus, 36310 Vigo, Spain. \\ \{javier,xil\}@det.uvigo.es. \\ ${ }^{\S}$ Dpto. Ingeniería Telemática, Universidad Carlos III de Madrid, Spain \\ \{jmoreno;acuevas\}@it.uc3m.es
}

\begin{abstract}
In a heterogeneous MANET, based on wireless LANs linked together by satellite, the overall channel efficiency is impaired by multiple effects, because of multipath fading in the terrestrial segment and atmospheric fading on the satellite link. In this paper we address this issue by applying forward erasure correction codes (FZC) to MPEG-4 video sequences exchanged by the hosts of a hybrid network, made of a satellite link and a wireless LAN using 802.11b devices. A standard video streaming application runs on one end of the satellite link while, at the other end, a wireless ad hoc network receives the multicast video stream. This work aims at demonstrating the improvement in Quality of Service (QoS) of the video transmitted in the hybrid network. The main parameters measured are the packet loss, the delivery delay, and the overhead in bandwidth occupancy imposed by the use of FZC. The received video is then evaluated by using a MOS (Mean Opinion Score) procedure.
\end{abstract}

\section{INTRODUCTION}

$\mathrm{n}$ the last years, the Mobile Ad hoc NETwork (MANET) Iparadigm has received considerable attention in the literature [1]. Generally speaking, available bandwidth is a precious resource in MANETs; saving bandwidth becomes thus a challenge that is dealt with by using dynamic control of allocated resources and the adoption of bandwidth-saving network protocols, i.e. multicast [2]. This consideration is also fundamental in hybrid networks, such as a combination of MANETs and infra-structured networks (including satellite links). The use of multicast protocols allows avoiding the waste of resources in satellite networks, especially in the context of streaming services. Protocols needing a return path to obtain a reliable communication,

Work funded by the European Commission in the framework of "SatNEx" NoE (contract no. 507052). such as TCP or Automatic Repeat Request (ARQ), are seldom used in such a context. Instead, forward error correction techniques are considered more useful for this purpose: the sender transmits some amount of redundant information, which allows the reconstruction of missing data at the receiver without further interactions with the transmitter. This approach generally simplifies both the sender and the receiver, since it reduces the time for recovering missing packets, often making unicast retransmissions and a feedback channel unnecessary. Some applications relying on multicast communication, e.g. audio or videoconferencing tools, can tolerate segment losses with relatively graceful degradation of performance. On the contrary, other applications, such as electronic whiteboards, electronic newspapers and software distribution have more strict requirements and need reliable delivery of all data segments. Thus, they would greatly benefit from high communication reliability.

In a heterogeneous MANET, based on wireless LANs linked together via satellite, the overall channel efficiency is impaired by multiple effects, because of multipath fading in the terrestrial segment and atmospheric fading on the satellite link. In this paper, we address this issue by applying forward erasure correction codes (FZC) to MPEG-4 video sequences exchanged by the hosts of a hybrid network. The network is made of a satellite link and a wireless LAN that uses 802.11 b devices. The stream is FZC encoded at the streamer and decoded at the individual receivers. Encoding and decoding operations occur just above the transport layer. This approach has the advantage of being independent of the equipment, the operating system and the end-user application. It performs as middleware between the application and the underlying operating system services, thus allowing the use of standard streaming applications both at the sender and the receiver. This work aims at demonstrating the 
improvement in quality of service of the video transmitted in the hybrid network. The main parameters measured are the packet loss, the delivery delay, and the overhead in bandwidth occupancy imposed by the use of FZC. The received video is then evaluated by using a MOS (Mean Opinion Score) procedure.

While the concept of using FZC has been widely studied for several years $[3,4]$, the literature is lacking in experimental results in a hybrid wireless environment. In the following, we first describe our test scenario and then we present measurements obtained in the described environment.

\section{The ReAl CASE StUdy}

The forward error correction technique employed in our experiments a class of linear block codes based on Vandermonde matrices [5]. Basically, $k$ blocks of source data are encoded to produce $n$ blocks of encoded data, such that any subset of $k$ encoded blocks suffices to reconstruct all source data (with $n>k$ ). Let $x$ be the vector of source blocks and $y$ the vector of encoded blocks. Then, the problem is to find an appropriate $n \times k$ coding matrix $C$ such that $y=C x$ and $x=C^{\prime-1} y^{\prime}$, for any subset $y^{\prime}$ of $k$ blocks in $y$, where $C^{\prime}$ is the corresponding row-wise sub-matrix in $C$ (a linear block code is said to be systematic if $I$ is a row-wise sub-matrix in $C$ ). As indicated in [5], the FZCs derived from Vandermonde coding matrices are of special interest; they employ efficient field arithmetic.

The video stream used in the experiment is coded using MPEG-4. MPEG-4 is the first standard that describes multimedia contents as a set of audio-visual objects to be presented, manipulated and transported individually. The high compression ratio and error resilience offered by MPEG-4 has driven an explosion of its popularity. The evaluation of FZC performance in MPEG-4 stream delivery on the single wireless path is not new. For example, in [6] the joint usage of FZCs and MPEG-4's Fine Granularity Scalability (FGS) - a highly suitable technique for IEEE 802.11 -is studied, while [7] studies the behavior of FZCs when applied to MPEG-4 streaming via $3 \mathrm{G}$ networks.

Anyway, there is a lack of experimental results in hybrid wireless environment. In order to provide such results, our target scenario is composed of a video-streaming server interconnected to an ad hoc wireless network through an OBP (On Board Processing) satellite. The satellite link is carried by the Eutelsat HotBird6 satellite in Ka band, and uses the technology known as Skyplex Data [8,9], which is an IPbased satellite network, derived from DVB-RCS. We used this satellite technology in a point-to-multipoint topology, by using a single multicast receiver. The Skyplex link we used has a bandwidth of $2 \mathrm{Mb} / \mathrm{s}$. Figure 1 depicts the scenario of our experiment. The video streamer transmits a FZC encoded multicast stream on the satellite; the local gateway receives the traffic from the satellite multicast receiver and forwards it to the wireless clients. The ad-hoc wireless LAN uses the IEEE $802.11 \mathrm{~b}$ standard [10].

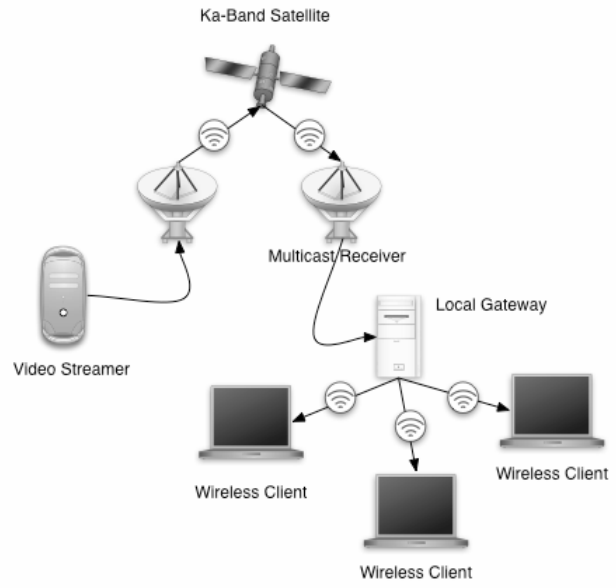

Fig. 1. The test-bed network topology

The mobile devices are IBM Thinkpad R40e laptops (Celeron 2.0 Ghz with $256 \mathrm{Mb}$ Ram running Debian Linux with a 2.6.8 kernel), and they are equipped with CNet CNWLC-811 IEEE $802.11 \mathrm{~b}$ wireless cards. The indoor environment is depicted in Fig. 2.

The dashed lines in Fig. 2 represent the shortest radio paths between the local gateway and the client devices A and B (black stars). The rooms are at the first floor of the building of the ISTI Institute in Pisa; they are delimited bhy thin walls.

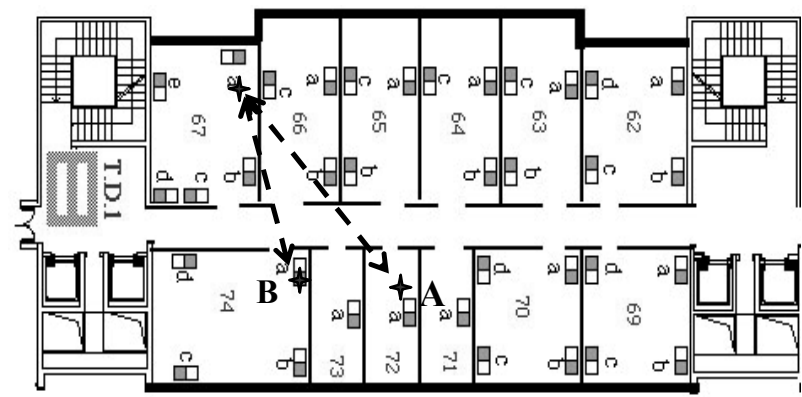

Fig.2 Plant of the building 


\section{EXPERIMENTAL RESULTS}

Notice that IEEE 802.11 includes the use of automatic repeat request (ARQ) [10] only in unicast mode. We implemented two software modules, an encoder and a decoder, written in C language, by using Rizzo's library [11]. The encoder works at the transport layer by fetching blocks of $k$ information packets from the video stream and then transmitting $k+l$ UDP packets ( $k$ of information $+l$ of redundancy) towards the receiving host. At the receiving host, the decoder fetches $k$ of the $k+l$ packets per block and recovers the information, provided that no more than $l$ packets are lost in a single block of packets. The receiver then feeds the VLC 0.8.1 decoder with the received stream. VLC uses a packet size of 1316 bytes, which is a block of 7 MPEG-4 frames. The encoder adds a preamble of 4 bytes for the sequence number, which is then cancelled by the decoder. The overall length of the 802.11 MSDU is then equal to $8+20+8+4+1316$ bytes, keeping into account the UDP, IP, and SNAP/LLC headers.

\section{A. Packet Error Rate}

The MPEG stream carries an Xvid version of the Pirates of Caribbean movie, with a frame rate of 25 frames per second at $576 \times 320$ pixels, and an average data stream of $939 \mathrm{~kb} / \mathrm{s}$. Our experiments show that the satellite channel is error free (thus the only problem is the intrinsic satellite delay), while the wireless channel is error prone. Thus, the error recovery aspect must be addressed in the wireless environment. By default, 802.11 devices use an internal algorithm for changing the transmission signal rate in order to adapt to varying channel conditions. Since our aim is to verify the performance of different coding schemes, we fixed the signal rate, by disabling the internal auto rate-change algorithm. We used two data bit rates: 11 and $5.5 \mathrm{Mb} / \mathrm{s}$. Lower transmission rates were not considered because the stream throughput would have exceeded the available information rate of the wireless channel. We analyze the channel between the local gateway and the A node (see Fig. 2), and between the local gateway and the B node (see Fig. 2), respectively. In the first case, which is the worst case (due to the distance between the gateway and the client A), the experiment in uncoded mode (no FZC) shows that packet loss can be reduced from $13 \%$ to about $6 \%$ by changing the transmission rate from 11 to 5.5 $\mathrm{Mb} / \mathrm{s}$. In this case, the channel occupancy increases by $56 \%$. A similar redundancy is required when using an FZC coding ratio of 150/100; however, in the latter case, we measured a much better performance, with a packet loss rate of $0.8 \%$. Better performance yet can be obtained by using an FZC with
$120 / 100$ coding ratio, which increases channel occupancy by only $20 \%$.

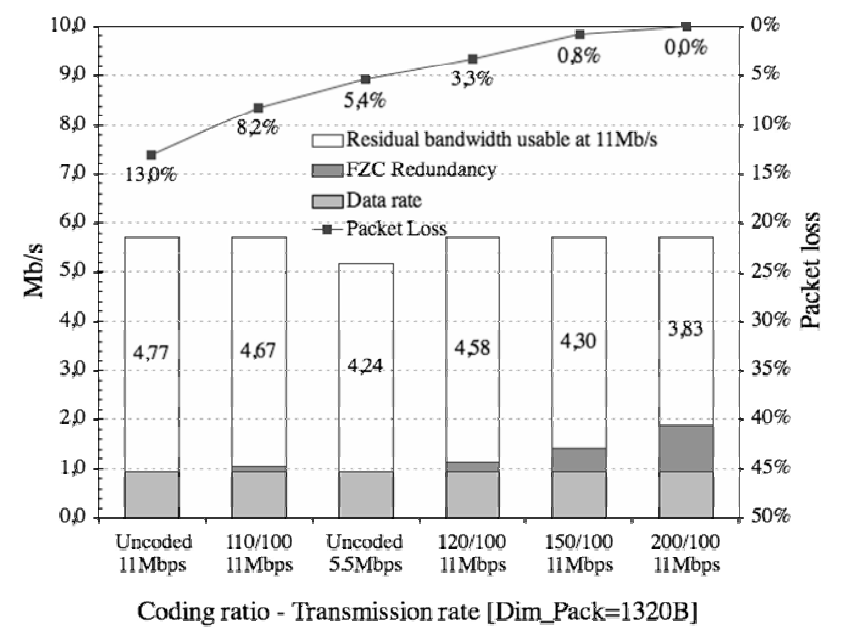

Fig. 3. Redundancy and error correction performance vs. coding ratio for client A.

When the B node is considered, the experiment in uncoded case (no FZC) shows that packet loss can be reduced from $5.9 \%$ to about $0.4 \%$ by changing the transmission rate from 11 to 5.5 $\mathrm{Mb} / \mathrm{s}$. However, if we use an FZC coding ratio of 110/100, we measured a much better performance (only $0.3 \%$ of packet loss) by increasing the channel occupancy by only $10 \%$. Choosing a coding ratio of 120/100, all packet losses (about 6\%) can be recovered by increasing the channel occupancy by $20 \%$.

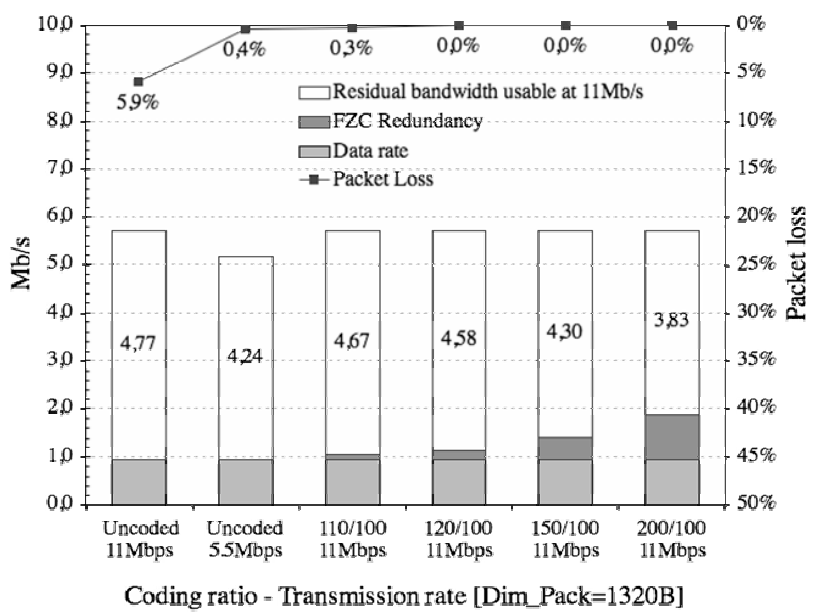

Fig. 4. Redundancy and error correction performance vs. coding ratio for client B

All measurements are summarized in Figures 3 and 4, where the residual bandwidth is defined as the bandwidth left available on the channel for use by other communications performed at 11 
$\mathrm{Mb} / \mathrm{s}$. Notice the case of $5.5 \mathrm{Mb} / \mathrm{s}$ where, while no FZC redundancy is introduced, the occupied channel share is $56 \%$ wider than the corresponding $11 \mathrm{Mb} / \mathrm{s}$ case, and the residual bandwidth is reduced accordingly.

Generally speaking, there is a tradeoff between redundancy and error correction performance; these results show that the usage of FZC is convenient with respect to changing the signal rate.

\section{B. Packet delivery delay}

A streaming application delivers time-based information, i.e. user data that has an intrinsic time component.

TABLE I.

MAXIMUM, MEAN AND VARIANCE OF DELIVERY DELAY FOR CLIENT A

\begin{tabular}{cccc}
\hline $\begin{array}{c}\text { Coding } \\
\text { ratio }\end{array}$ & $\begin{array}{c}\text { Max } \\
{[\mathrm{ms}]}\end{array}$ & $\begin{array}{c}\text { Mean } \\
{[\mathrm{ms}]}\end{array}$ & $\begin{array}{c}\text { Var. } \\
{[\mathrm{ms}]}\end{array}$ \\
\hline $110 / 100$ & 105.6 & 52.149 & 0.769 \\
$120 / 100$ & 115.2 & 54.99 & 0.792 \\
$130 / 100$ & 124.8 & 52.637 & 0.805 \\
$200 / 100$ & 192 & 53.805 & 1.675 \\
\hline
\end{tabular}

TABLE II.

\begin{tabular}{cccc}
\multicolumn{4}{c}{ MAXIMUM, MEAN AND VARIANCE OF DELIVERY DELAY FOR CLIENT B } \\
\hline $\begin{array}{c}\text { Coding } \\
\text { ratio }\end{array}$ & $\begin{array}{c}\text { Max } \\
{[\mathrm{ms}]}\end{array}$ & $\begin{array}{c}\text { Mean } \\
{[\mathrm{ms}]}\end{array}$ & $\begin{array}{c}\text { Var. } \\
{[\mathrm{ms}]}\end{array}$ \\
\hline $110 / 100$ & 105.6 & 48.522 & 0.796 \\
$120 / 100$ & 115.2 & 48.639 & 0.824 \\
$130 / 100$ & 124.8 & 47.992 & 0.715 \\
$200 / 100$ & 192 & 53.585 & 1.681 \\
\hline \hline
\end{tabular}

By using erasure codes we introduce a packet delivery delay. In fact, when $h$ packets in a block are lost, we must wait for all $k$ packets of information plus $h$ of redundancy. Delivery delay is an important parameter that must be evaluated like the packet loss. Our experiments show that the mean delivery delay for the node $\mathrm{A}$ is about $50 \mathrm{~ms}$, and it is less than the delivery delay for the node B (48 ms). This is due to the fact that node $\mathrm{A}$ has a greater number of packet losses than node B. The maximum packet delivery delay is evaluated as the time necessary to recovery all the information when a number of packets equal to the redundancy packets is lost. Tables I and II show the packet delivery delay for clients $\mathrm{A}$ and $\mathrm{B}$, respectively.

\section{Mean opinion Score (MOS)}

The MOS is the most widely known video quality metric. MOS is a subjective score, ranging from 5 (Excellent) down to 0 (Unacceptable). Thirty persons have answered to three quality questions (overall, video, and audio quality) for each coding ratio of the video received by client $\mathrm{A}$; mean opinion scores have been calculated. The results for each quality question are shown in Fig. 5.

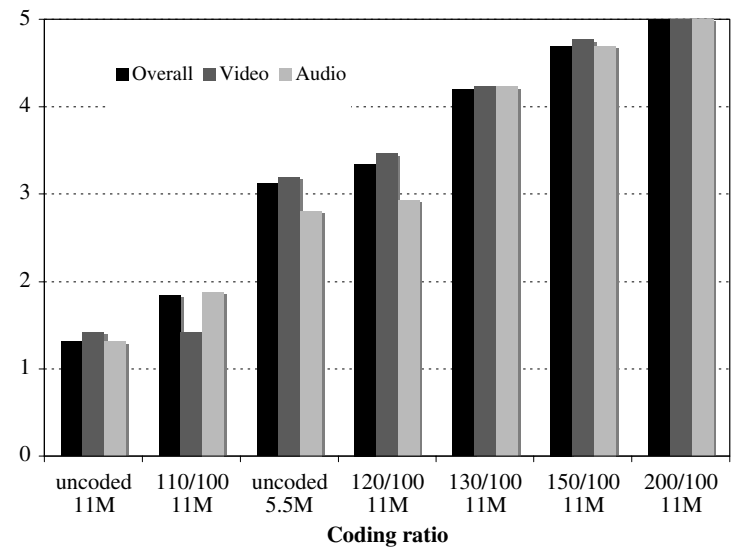

Fig. 5. MOS of received video by client A.

It can be seen that the coding ratio is important for opinions on the quality; this is true for all three questions. Not surprisingly, the best video quality is obtained by using the 200/100 coding ratio. When the coding ratio decreases from 200/100 to the uncoded case, the quality ratings become progressively poorer.

TABLE III.

ACCEPTABILITY OF RECEIVED VIDEO BY CLIENT A

\begin{tabular}{ccccccc}
\hline \hline Uncoded & $110 / 100$ & Uncoded & $120 / 100$ & $130 / 100$ & $150 / 100$ & $200 / 100$ \\
$11 \mathrm{M}$ & $11 \mathrm{M}$ & $5.5 \mathrm{M}$ & $11 \mathrm{M}$ & $11 \mathrm{M}$ & $11 \mathrm{M}$ & $11 \mathrm{M}$ \\
\hline $7,7 \%$ & $15,4 \%$ & $26,9 \%$ & $34,6 \%$ & $100,0 \%$ & $100,0 \%$ & $100,0 \%$ \\
\hline
\end{tabular}

Acceptability opinions for each test were based on a binary choice: acceptable or not acceptable. Figure 6 displays the percentage of persons who report 'acceptable' opinions for each coding ratio. Table III clearly shows that the video quality is acceptable when using a coding ratio greater than 130/100.

Video applications are sensitive to packet losses and, even if a few packets are lost, the video streaming performance can be considered unacceptable: with $3.3 \%$ of packet loss (coding ratio of $120 / 100$ ), only $30 \%$ of subjects found the performance acceptable. Some samples of the proposed video for the MOS are depicted in fig 6 . 


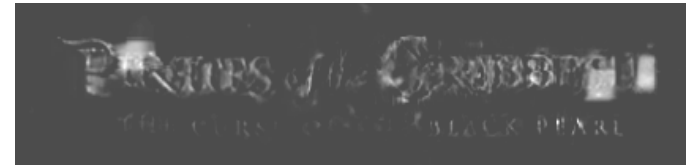

Uncoded@11Mbps - Packet Loss 13.0\%

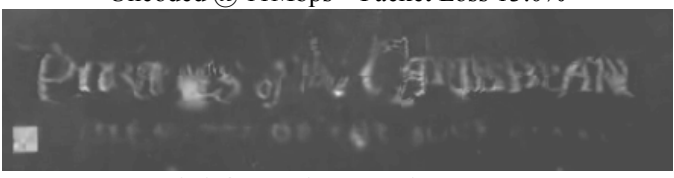

Uncoded @ 5.5Mbps - Packet Loss 5.4\%

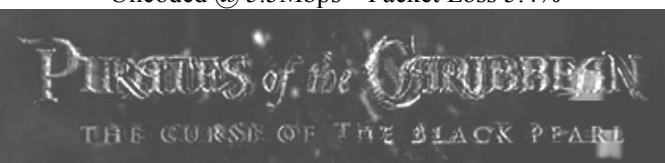

FZC 110/100@11Mbps-Packet Loss 8.2\%

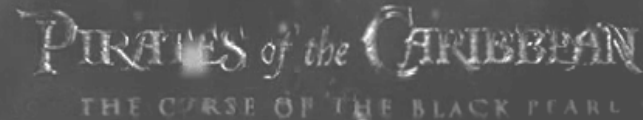

FZC 120/100@11Mbps - Packet Loss 3.3\%

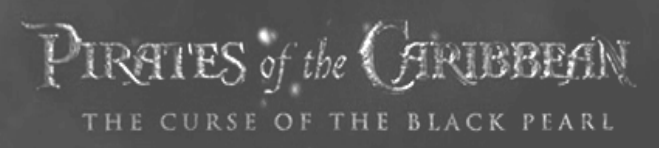

FZC 150/100@11Mbps - Packet Loss 0.8\%

Fig. 6 Samples of the streamed video trailer at different coding and bit rates

\section{CONCLUSION AND CONSIDERATIONS}

In this paper we experimented with the use of FZC codes for supporting a reliable video streaming service in a WLAN connected via satellite to a multicast streaming server. This testbed matches professional platforms used for e-learning and lectures over vast areas. Measurements show that using FZC is a flexible method for compensating for errors on the terrestrial segment. The delay measured in the experiment is about $50 \mathrm{~ms}$ on the average, and always less than $200 \mathrm{~ms}$. The performance of FZC is better than lowering the code rate from $11 \mathrm{Mb} / \mathrm{s}$ to $5.5 \mathrm{Mb} / \mathrm{s}$. Future work will examine a wider set of environmental conditions - both in the satellite and terrestrial segments, of transmission bit rates and of FZC algorithms.

\section{ACKNOWLEDGEMENTS}

The authors want to thank Mr. Antonio Blasco Bonito for his invaluable help in the setup of the satellite and the wireless networks.

\section{REFERENCES}

[1] Aschenbruck, N.; Frank, M.; Martini, P.; Tolle, J., "Human mobility in MANET disaster area simulation - a realistic approach" Local Computer Networks, 2004. 29th Annual IEEE International Conference on , 16-18 Nov. 2004 Pages:668 - 675

[2] C. de Morais Cordeiro, H. Gossain, D.P. Agrawal, "Multicast over wireless mobile ad hoc networks: present and future directions", IEEE Network, Jan/Feb 2003.

[3] L. Rizzo and L. Vicisano. "RMDP: an FEC-based reliable multicast protocol for wireless environments". Mobile Computer and Communication Review, 2(2), April 1998

[4] Zorzi, M: "Performance of FEC and ARO Error control in bursty channels under delay constraints", VTC'98, Ottawa, Canada, May 1998

[5] L. Rizzo, "Effective erasure codes for reliable computer communication protocols", ACM Computer Communication Review, April 1997.

[6] T. P. Chen and T. Chen, "Fine-Grained Rate Shaping for Video Streaming over Wireless Networks", IEEE International Conference on Acoustics, Speech and Signal Processing (ICASSP-03), Hong Kong, 2003

[7] V. Stankovic, R. Hamzaoui and Z. Xiong, "Live Video Streaming over Packet Networks and Wireless Channels", Packet Video 2003, Nantes, France, 2003.

[8] E. Feltrin, E. Weller, E. Martin, K. Zamani, "Implementation of a satellite network based on Skyplex technology in Ka band", Ninth Ka and Broadband Communications Conference, Ischia, Italy, November 2003.

[9] http://www.eutelsat.com/fr/satellites/pdf/dealers/ annex_d_skyplex.pdf

[10] IEEE Std 802.11b-1999 Supplement to IEEE Standard for Information technology- Telecommunications and information exchange between systems- Local and metropolitan area networks- Specific requirements Part 11: Wireless LAN Medium Access Control (MAC) and Physical Layer (PHY) Specifications: Higher-speed Physical Layer Extension in the 2.4 GHz Band.

[11] Luigi Rizzo's page on FEC at <http://info.iet.unipi.it/ luigi/fec.html>. 